\title{
The pro-apoptotic activity of Drosophila Rbf1 involves dE2F2-dependent downregulation of diap1 and buffy mRNA
}

\author{
A Clavier ${ }^{1}$, A Baillet ${ }^{1}$, A Rincheval-Arnold ${ }^{1}$, A Coléno-Costes ${ }^{2,3,4}$, C Lasbleiz ${ }^{1,5}$, B Mignotte $^{1}$ and I Guénal ${ }^{*, 1}$
}

The retinoblastoma gene, $r b$, ensures at least its tumor suppressor function by inhibiting cell proliferation. Its role in apoptosis is more complex and less described than its role in cell cycle regulation. Rbf1, the Drosophila homolog of Rb, has been found to be pro-apoptotic in proliferative tissue. However, the way it induces apoptosis at the molecular level is still unknown. To decipher this mechanism, we induced rbf1 expression in wing proliferative tissue. We found that Rbf1-induced apoptosis depends on dE2F2/dDP heterodimer, whereas dE2F1 transcriptional activity is not required. Furthermore, we highlight that Rbf1 and dE2F2 downregulate two major anti-apoptotic genes in Drosophila: buffy, an anti-apoptotic member of Bcl-2 family and diap1, a gene encoding a caspase inhibitor. On the one hand, Rbf1/dE2F2 repress buffy at the transcriptional level, which contributes to cell death. On the other hand, Rbf1 and dE2F2 upregulate how expression. How is a RNA binding protein involved in diap1 mRNA degradation. By this way, Rbf1 downregulates diap1 at a post-transcriptional level. Moreover, we show that the dREAM complex has a part in these transcriptional regulations. Taken together, these data show that Rbf1, in cooperation with dE2F2 and some members of the dREAM complex, can downregulate the anti-apoptotic genes buffy and diap1, and thus promote cell death in a proliferative tissue.

Cell Death and Disease (2014) 5, e1405; doi:10.1038/cddis.2014.372; published online 4 September 2014

The retinoblastoma gene $(r b)$ is the first tumor suppressor identified in human cells. Its product, $\mathrm{pRb}$, inhibits cell proliferation by controlling the G1/S transition. ${ }^{1}$ The best characterized partners of $\mathrm{pRB}$ in cell cycle regulation belong to the E2F family of transcription factors. ${ }^{2}$ In contrast to its tumor suppressor role, $\mathrm{pRb}$ is surprisingly often described as an anti-apoptotic protein. ${ }^{3-10}$ However, a growing number of studies show a pro-apoptotic role for pRb. ${ }^{11-14}$ Drosophila, which presents a lesser genetic complexity than mammals, offers the possibility to decipher the roles of Rb and E2F family proteins in apoptosis in vivo. Indeed, the Drosophila genome contains two E2F genes ${ }^{15-17}$ (de2f1 and de2f2), one DP gene $^{15}(d D p)$ that encodes an E2F cofactor and two $R b$ genes $^{18,19}$ ( $r b f 1$ and $\left.r b f 2\right)$. dE2F1 acts mostly as a transcriptional activator, ${ }^{20}$ whereas $\mathrm{dE} 2 \mathrm{~F} 2$ represses transcription. ${ }^{21}$ As $\mathrm{pRb}$ protein in mammals, Rbf1 can bind both activator and repressor E2F members. ${ }^{21}$ Furthermore, it fulfills the same function as $\mathrm{pRb}$ in the cell cycle. Rbf1 is thus considered as a $\mathrm{pRb}$ homolog. rbf1 loss-of-function is lethal at early larval stage $^{20,22}$ that attests its essential role. Homozygous rbf1mutant embryos have many apoptotic cells ${ }^{22}$ and it is admitted that Rbf1 protects cells by inhibiting the transcriptional activity of $\mathrm{dE} 2 \mathrm{~F} 1$ that is considered as a pro-apoptotic factor. Indeed, de2f1 and $d D p$ co-expression induces apoptosis in the eye imaginal discs ${ }^{23}$ and the pro-apoptotic genes reaper and dark are dE2F1-transcriptional targets. ${ }^{24,25}$ In contrast to these data, we have previously shown that $r b f 1$ can also have a proapoptotic function. Indeed, rbf1 overexpression in proliferating cells of wing imaginal discs leads to apoptosis and loss of tissue in adult wings. ${ }^{26}$ This cell death is caspase dependent and can be inhibited by de2f1 expression. However, the precise mechanism underlying rbf1-induced apoptosis is still unknown. Rbf1 being mainly described as a transcriptional regulator, one may wonder whether this activity is involved in its pro-apoptotic effect. Recent reports have clarified the role of Rbf1 in transcription. Rbf1 binding to chromatin requires dE2F/dDP complexes. ${ }^{27}$ This binding is mainly observed near transcription start sites (TSS). When associated with dE2F2, Rbf1 belongs to a transcription regulator complex named dREAM $^{28-30}$ (drosophila RBF, E2F and Myb-interacting proteins). This complex maintains the transcriptional repression of certain E2F target genes in the proliferating tissues by at least two distinct mechanisms: histone deacetylation of nucleosomes near TSSs and dimethylation of histone $\mathrm{H} 3$ Lys27 at nucleosomes located downstream of TSSs. ${ }^{31}$ Although the first reports described the dREAM complex as

\footnotetext{
${ }^{1}$ Laboratoire de Génétique et Biologie Cellulaire, EA4589, Université de Versailles Saint-Quentin-en-Yvelines, Ecole Pratique des Hautes Etudes, 2 avenue de la Source de la Bièvre, Montigny-le-Bretonneux, France; ${ }^{2}$ Laboratoire de Biologie du Développement-Institut de Biologie Paris Seine, Sorbonne Universités, UPMC Univ Paris 06, UMR7622, Paris, France and ${ }^{3}$ CNRS, UMR7622, Laboratoire de Biologie du Développement-Institut de Biologie Paris Seine, Paris, France

${ }^{*}$ Corresponding author: I Guénal, Laboratoire de Génétique et Biologie Cellulaire, EA4589, Université de Versailles Saint-Quentin-en-Yvelines, Ecole Pratique des Hautes Etudes, 2 avenue de la Source de la Bièvre 78180 Montigny-le-Bretonneux, France. Tel: +33 0170429436 ; Fax: +33 0170429503 ; E-mail: isabelle.guenal@uvsq.fr

${ }^{4}$ Present address: IGH—CNRS, 141 rue de la Cardonille, 34396 Montpellier, France.

${ }^{5}$ Present address: UMR Inserm U710-UM2-EPHE, Université Montpellier 2, place Eugene Bataillon-CC105, 34095 Montpellier, France.

Abbreviations: ChIP, chromatin immunoprecipitation; dIAP1, Drosophila inhibitor of apoptosis; dREAM, drosophila RBF, E2F and Myb-interacting proteins; en, engrailed; how, held out wings; $r b$, retinoblastoma gene; TSS, transcription start sites; vg, vestigial

Received 07.3.14; revised 23.7.14; accepted 28.7.14; Edited by E Baehrecke
} 
an exclusive transcriptional repressor, the recent ones showed that this complex is also required to maintain the expression of some genes, highlighting that it can also participate in transcriptional activation. ${ }^{32}$ Several screens have identified Rbf1 target genes. ${ }^{27,32,33}$ However, how the regulation of these genes is related to the different functions of Rbf1 remains to be explained. Notably, the targets of Rbf1 in apoptosis are not known.

Here we show that Rbf1-induced apoptosis results from transcriptional regulation of at least two genes by Rbf1 and dE2F2. First, Rbf1 and dE2F2 repress the expression of buffy, the anti-apoptotic member of the Bcl-2 family in Drosophila. Second, Rbf1 and dE2F2 activate the expression of how (held out wings), which encodes an RNA-binding protein that destabilizes diap1 (Drosophila inhibitor of apoptosis).

\section{Result}

dE2F2 and dDP cooperate with Rbf1 to induce apoptosis. As previously described, overexpression of rbf1 in the dorsal region of wing imaginal discs using the UAS-Gal4 system with the 'vestigial' (vg) Gal4 driver induced notches along the wing margin. The number of notches correlated with the amount of apoptosis in wing imaginal discs of third instar larvae. ${ }^{26}$ To determine the relative importance of the two dE2F factors in Rbf1-induced apoptosis, we performed genetic interaction tests. For each gene studied, we verified that the alteration of this gene expression level by itself (overexpression, RNAi or mutant) did not induce any wing phenotype, nor apoptosis at larval stage. Wing phenotypes were classified into four categories according to the number of notches: wild type (no notch), weak, intermediate and strong (Figure 1a). Notches were counted in the wings of flies overexpressing rbf1 in a heterozygous dE2F2-mutant background (vg-Gal4>UAS-Rbf1; dE2F2 $2^{76 Q 1} /+$ ) and in flies simultaneously overexpressing rbf1 and de2f2 (vgGal4> UAS-rbf1; UAS-dE2F2) (Figure 1b). When rbf1 was overexpressed in $d E 2 F 2^{76 Q 1}$ heterozygous context, a significant shift of the distribution toward weaker phenotypes was observed as compared with overexpression of rbf1 alone (Figure 1b). On the contrary, when rbf1 and dE2F2 were co-overexpressed, the distribution significantly shifted toward stronger phenotypes. Thus, these results show that dE2F2 is necessary for Rbf1-induced notched wing phenotype. Previous data have shown that de2f1 heterozygous loss-of-function mutant context enhances rbf1-induced a

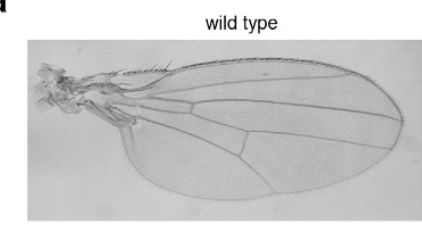

b
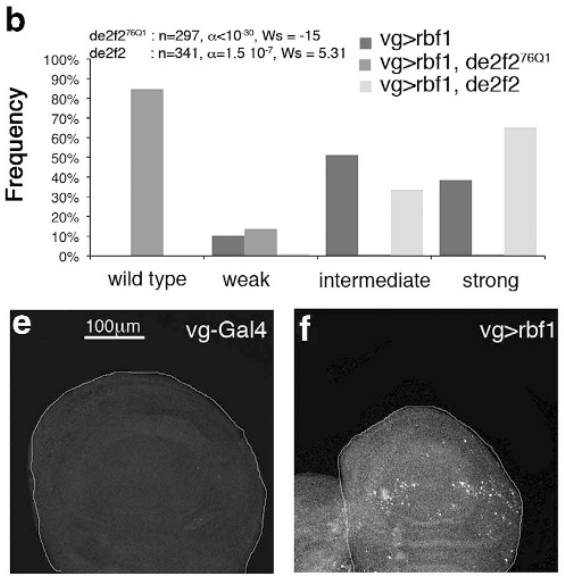

k

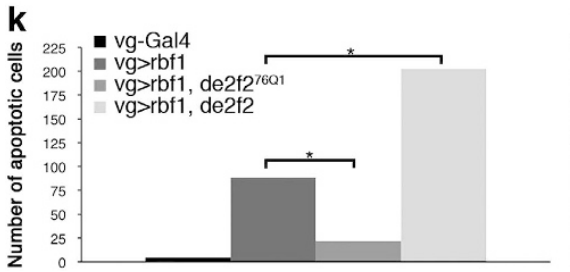

weak

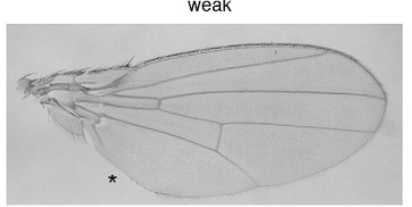

C
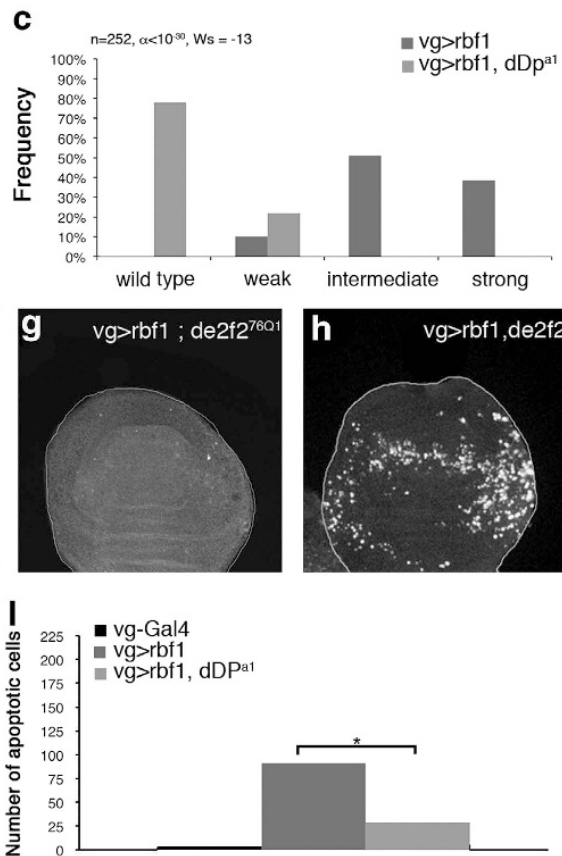

intermediate

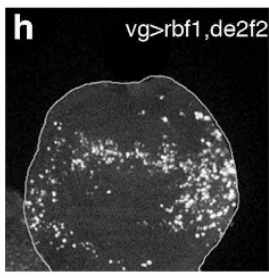

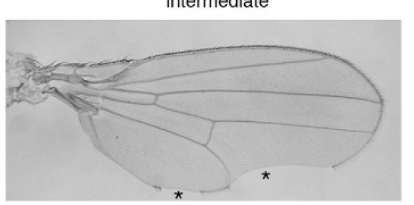

d
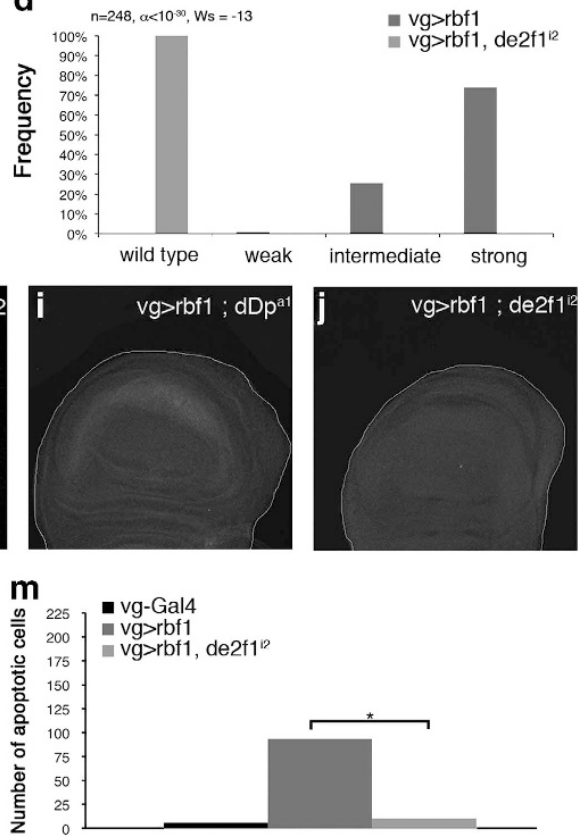

Figure 1 Rbf1-induced apoptosis involves dE2F2 and dDP. (a) Wing phenotypes were grouped in four categories (wild type, weak, intermediate and strong) according to the number of notches observed on the wing margin (asterisks). (b-d) Distribution of notch wing phenotypes in vg-Gal4 $>$ UAS-rbf1, vg-Gal4 $>$ UAS-rbf1; de2f2 ${ }^{76 Q 1}$ and $v g$-Gal4 > UAS-rbf1, UAS-de2f2 (b), in vg-Gal4>UAS-rbf1 and vg-Gal4>UAS-rbf1; dDp ${ }^{a 1}$ (c), and in vg-Gal4>UAS-rbf1 and vg-Gal4>UAS-rbf1; de2f1' (d). Statistical analysis was performed using Wilcoxon tests. Each experiment was independently performed three times; as the results were similar, only one experiment is presented here. (e-j) Apoptotic cells were visualized by TUNEL staining (white dots) of wing imaginal discs of the genotype indicated at the top of the image. All the pictures are at the same scale, scale bar: $100 \mu \mathrm{m}$. (k-m) Quantification of TUNEL-positive cells in the wing pouch. Asterisks indicate a statistically significant difference between two genotypes (Student's $t$-test, $P<0.05)$ 
notched wing phenotype, showing that de2f1 antagonizes rbf1-induced phenotypes. ${ }^{26}$ These results suggest that $\mathrm{dE} 2 \mathrm{~F} 1$ and dE2F2 have antagonistic roles in rbf1-induced notched wing phenotype. $\mathrm{dDP}$ is the cofactor shared by dE2F1 and dE2F2. In the absence of dDP, both dE2F1 and dE2F2 transcriptional activity is abolished. ${ }^{34}$ In $d D P^{a 1}$ heterozygote context, the rbf1-induced notched wing phenotypes significantly shifted toward weaker phenotypes (Figure 1c) as observed in dE2F2-mutant context. This result indicates that the reduction of the net transcriptional activity of $d E 2 F$ factors due to $d D p$-mutant context rescues rbf1-induced loss of tissue. Thus, dE2F's net transcriptional activity promotes rbf1-induced notched wing phenotype.

We used de2f1' $1^{i 2}$ mutant to determine whether dE2F1 inhibitory role in rbf1-induced loss of tissue involved its transactivation domain. This dE2F1 mutant lacks both the transactivation domain and its ability to bind Rbf1. ${ }^{20}$ However, it retains the DNA binding domain. By this way, it is able to bind dE2F consensus site and could exclude some complexes, such as dE2F2/Rbf1, from these genomic sites. ${ }^{35}$ When rbf1 was overexpressed in a de $2 f 1^{i 2}$ heterozygous context, the distribution of the phenotypes shifted toward weaker phenotypes as compared with the overexpression of rbf1 alone (Figure 1d). Thus, dE2F $1^{\text {i2 }}$ suppresses rbf1-induced loss of tissue and the transcriptional activation mediated by dE2F1 does not seem to be required to inhibit rbf1-induced loss of tissue.

To verify that the variation of the phenotypic distribution between these different genetic contexts correspond to a variation of the amount of apoptosis in larvae, we performed TUNEL staining of third instar larval wing imaginal discs. Few apoptotic cells were detected in $\mathrm{vg}$-Gal4/+ control (Figure 1e). On the contrary, many cells were TUNEL labeled in $v g-G a l 4 /+; U A S-r b f 1 /+$ wing discs (Figure 1f). When rbf1 was overexpressed in a de2f2 $2^{76 Q 1}, d D p^{a 1}$ or de2f $1^{i 2}$ context (Figures 1g-j), we observed a significant decrease of TUNELlabeled cells as compared with the overexpression of rbf1 alone (Figures $1 \mathrm{k}-\mathrm{m}$ ). On the contrary, when rbf1 and de2f2 were co-overexpressed, the number of apoptotic cells was significantly increased (Figures $1 \mathrm{~h}$ and $\mathrm{k}$ ).

These data show that dE2F1 might inhibit Rbf1-induced apoptosis independently of its transactivation activity, whereas dE2F2 and dDP cooperate with Rbf1 to induce apoptosis in the wing imaginal disc, a proliferating tissue.

Rbf1 and dE2F2 induce a reduction of buffy and diap1 mRNA levels. dE2F2 is a well-known transcriptional repressor. To explain its role in rbf1-induced apoptosis, we hypothesized that an Rbf1/dE2F2 complex could repress anti-apoptotic genes, leading to cell death. We focused on the two bestdescribed anti-apoptotic factors in Drosophila, diap1 and buffy, which encode a caspase inhibitor and a member of the Bcl-2 family, respectively. When rbf1 was overexpressed under $v g$ control, buffy mRNA were significantly decreased as compared with the control $v g$-Gal4/+ (Figure 2a). In contrast, when rbf1 was inactivated by RNAi (vg-Gal4>UAS-RNAi-rbf1), buffy mRNA was significantly increased as compared with the control (Figure $2 b$ ). The same result was obtained in $d D P$ heterozygous loss-of-function context (data not shown). In $d E 2 F 2^{76 Q 1}$ heterozygous larvae, the amount of buffy mRNA was similar to the one of the control $v g-G a / 4 /+$. When rbf1 was overexpressed in a dE2F2 ${ }^{76 Q 1}$ heterozygous context, the amount of buffy mRNA was similar to the one of the $\mathrm{vg}-\mathrm{Ga} / 4 /+$ control (Figure 2a), indicating that $d E 2 F 2$ is necessary for rbf1-induced buffy mRNA decrease. These data suggest that Rbf1 represses buffy in wing imaginal discs in a de2f2-dependent manner. Similarly, rbf1 overexpression induced a decrease of diap1 mRNA level dependent of dE2F2 (Figure 2c). We did not observe any modification of diap1 mRNA levels when rbf1 was inactivated by RNAi (data not shown).

To verify whether the amount of buffy and diap1 mRNA correlated with rbf1-induced apoptosis, we performed genetic interaction tests. When rbf1 was overexpressed in a buffy ${ }^{H 37}$ heterozygous context, distribution of the wing phenotypes shifted toward stronger phenotypes as compared with the overexpression of rbf1 alone (Figure 2d). On the contrary, when rbf1 and buffy were co-overexpressed, distribution of the wing phenotypes shifted toward weaker phenotypes. The variation of the phenotypic distribution between these different genetic contexts correlated with a variation of the amount of apoptosis in wing imaginal discs (Figure 2f). These results suggest that the decrease of buffy mRNA is a part of the cell death mechanism induced by Rbf1.

When rbf1 was overexpressed and diap1 was simultaneously inactivated by RNAi, we observed an increase of notches in the wings (Figure $2 \mathrm{e}$ ) as well as an increase in the amount of apoptosis in wing imaginal discs (Figure $2 \mathrm{~g}$ ) as compared with the overexpression of rbf1 alone. On the contrary, we detected a phenotypic rescue when rbf1 and diap1 were co-overexpressed. This suggests that diap1 mRNA level reduction contributes to Rbf1-induced apoptosis.

Rbf1 and dE2F2 increase how mRNA leading to diap1 mRNA destabilization. Our data suggest that Rbf1 and dE2F2 could directly repress the transcription of diap1 and buffy. A putative E2F binding site is present in buffy $5^{\prime} \mathrm{UTR}$ but absent in diap1 (data not shown). To confirm that diap1 mRNA reduction was due to transcriptional regulation, we used a diap1-LacZ reporter transgene in en-Gal4/+ control wing imaginal discs and en-Gal4; UAS-rbf1 wing imaginal discs. In posterior compartment, rbf1 overexpression slightly alter the $\beta$-Gal staining aspect as compared with the en-gal4/+ control (Figures $3 a$ and $b$ ), probably due to the presence of apoptotic cells, but we could not observe a real staining decrease. Nevertheless, Diap1 protein had decreased in the posterior compartment upon rbf1 overexpression (Figure 3d). These data suggest that $r b f 1$ overexpression would induce a post-transcriptional reduction of diap1 mRNA.

Interestingly, it has been reported that diap1 is a target of How, an RNA-binding protein that belongs to the STAR family. ${ }^{36}$ Two How isoforms have been described. The short isoform, $\operatorname{How}(\mathrm{S})$, is involved in mRNA stability and splicing. ${ }^{37}$ The long isoform, How(L), binds the $3^{\prime} U T R$ of target mRNAs, leading to their destabilization and their rapid degradation. diap1 is a target of How $(\mathrm{L}) .{ }^{36}$ We used how ${ }^{\text {stru }}$ loss-of-function mutant to determine the implication of How in Rbf1-induced apoptosis. When rbf1 was overexpressed in a how stru heterozygous context, distribution of the phenotypes significantly shifted toward weaker phenotypes as compared with the expression of rbf1 alone. Consistently, the number of 
a
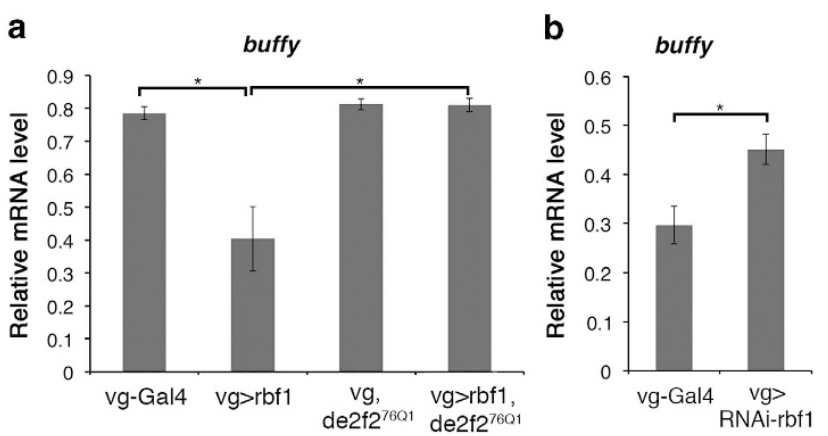

d

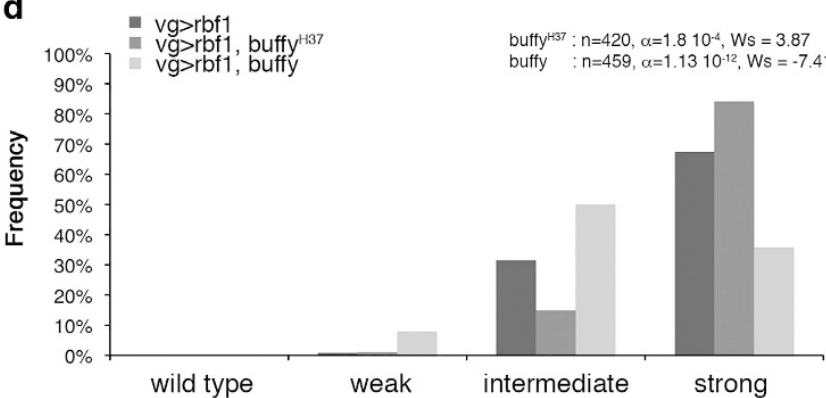

f

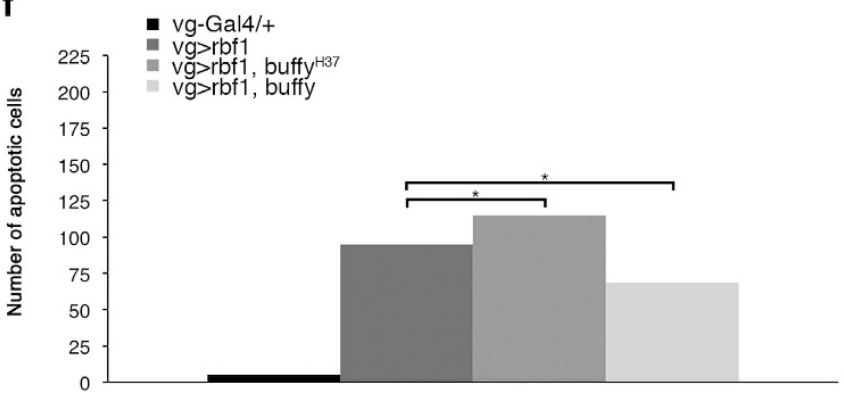

C

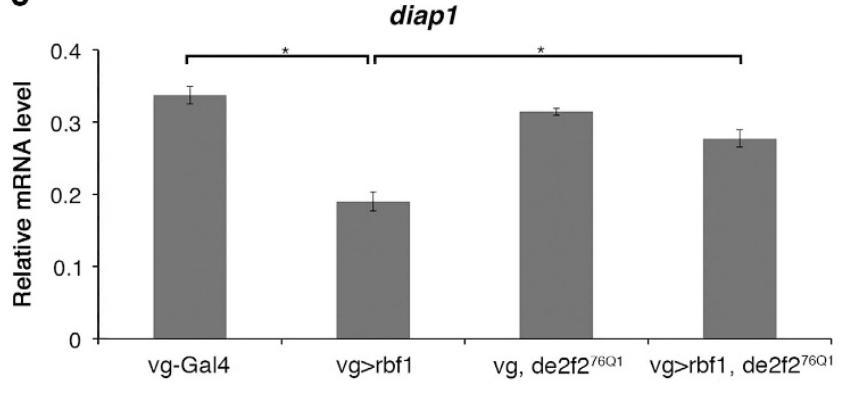

e

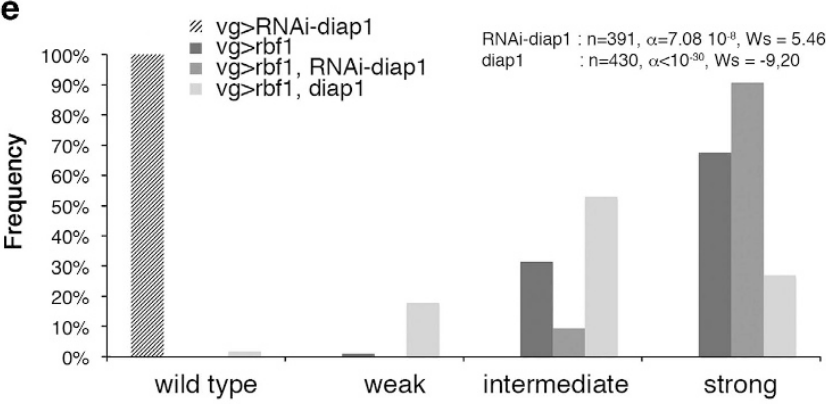

g

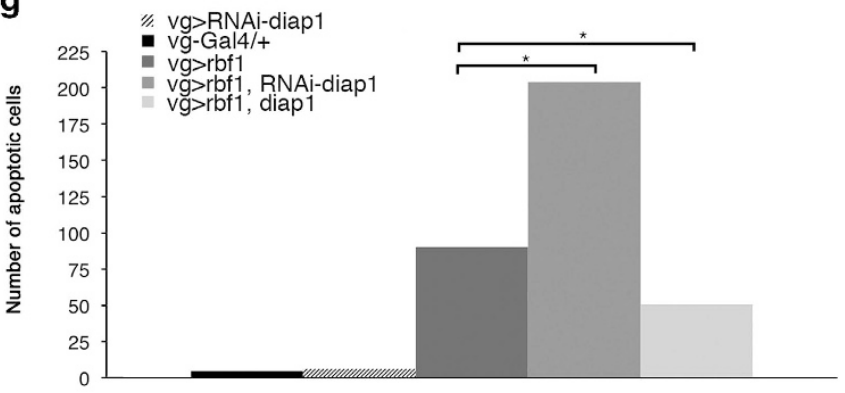

Figure 2 rbf1 overexpression induces downregulation of buffy and diap1 mRNA. (a-c) Quantification of buffy (a, b) and diap1 (c) mRNA by RT-qPCR in wing imaginal discs. Data are normalized against $r p 49$ and correspond to the mean of three independent experiments. Error bars are the S.E.M. Asterisks indicate statistical significant difference between two genotypes (Student's t-test, $P<0,05$ ). (d, e) Distribution of notches in wings of $v g$-Gal4 $>U A S$-rbf1, vg-Gal4 $>U A S$-rbf1; buffy ${ }^{H 37}$ and $v g$-Gal4 > UAS-rbf1; UAS-buffy flies (d), vg-Gal4> UAS-rbf1, vg-Gal4> UAS-rbf1; UAS-RNAi-diap1 and vg-Gal4> UAS-rbf1; UAS-diap1 flies (e). Wing phenotypes were grouped in four categories according to the number of notches (wild type, weak, intermediate, strong). Statistical analysis was performed using Wilcoxon tests. Each experiment was independently performed three times; as the results were similar, only one experiment is presented here. (f, g) Quantification of TUNEL-positive cells in the wing pouch. Asterisks indicate a statistically significant difference between two genotypes (Student's t-test, $P<0.05$ )

apoptotic cells decreased in wing imaginal discs of the same genotype (Figures $3 e$ and f). Thus, How is, necessary for Rbf1-induced apoptosis. To confirm that How is involved in diap1 regulation, we performed RT-qPCR. In how stru heterozygotes, diap1 mRNA were slightly but significantly decreased indicating that How is required to maintain a basal level of diap1 mRNA (Figure 3g). When rbf1 was overexpressed in a how ${ }^{\text {stru }}$ heterozygous context, diap1 mRNA increased as compared with the rbf1 overexpression alone and even exceeded the level observed in $\mathrm{vg}-\mathrm{Gal} / \mathrm{f}+\mathrm{c}$ control. Therefore, these data suggest that Rbf1-induced apoptosis leads to diap 1 mRNA destabilization by How.

To confirm whether diap1 downregulation by How in Rbf1induced apoptosis involved diap1 $3^{\prime}$ UTR, we performed a genetic interaction test using a UAS-diap 1 UY1835 transgenic line. This line bears a $P$ element in the $5^{\prime}$ UTR sequence of the diap1 gene, which allows overexpressing diap1 with its $3^{\prime}$ UTR sequence. ${ }^{38}$ Contrary to the phenotypic rescue observed when rbf1 was co-overexpressed with diap1 devoid of its $3^{\prime} U T R$ sequence (Figure 2e), rbf1co-overexpression with diap $1^{U Y 1835}$ did not lead to a rescue of notch phenotypes (Figure 3h). Interestingly, diap $1^{\text {UY1835 }}$ allowed an effective increase in the Diap1 protein level as attested by Diap1 immunostaining (Figure 3j); however, this protein level was significantly lower when rbf1 was overexpressed (Figure 3k). This suggests that Diap1 cannot accumulate in an rbf1-overexpressing context. When rbf1 and diap 1 UY1835 were co-overexpressed in a how ${ }^{\text {stru }}$ heterozygous context, the Diap1 protein accumulated (Figure 3I) and consistently, we observed a significant rescue as compared with the expression of rbf1 in a how ${ }^{\text {stru }}$ mutant context (Figure 3h, Wilcoxon test: $\left.n=308, \alpha<10^{-30}, \mathrm{Ws}=-8.95\right)$. The variation of the phenotypic distribution between these different genetic contexts correlated with a variation of the amount of apoptosis in wing imaginal discs (Figure $3 \mathrm{~m}$ ). Thus, the how ${ }^{\text {stru }}$ heterozygote context prevents diap 1 repression by 
a

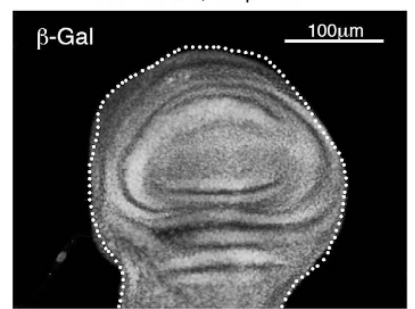

e

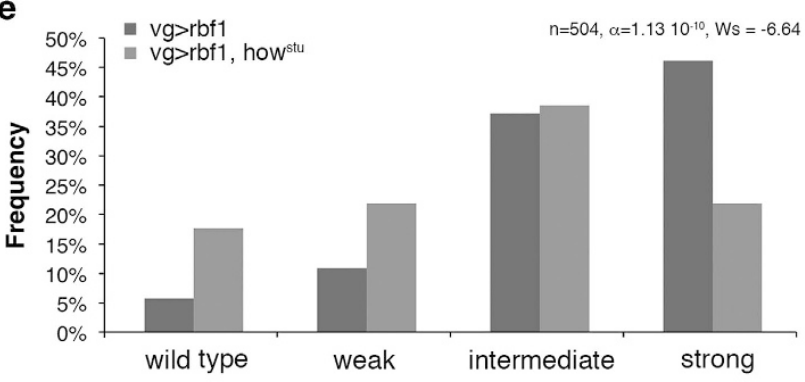

g
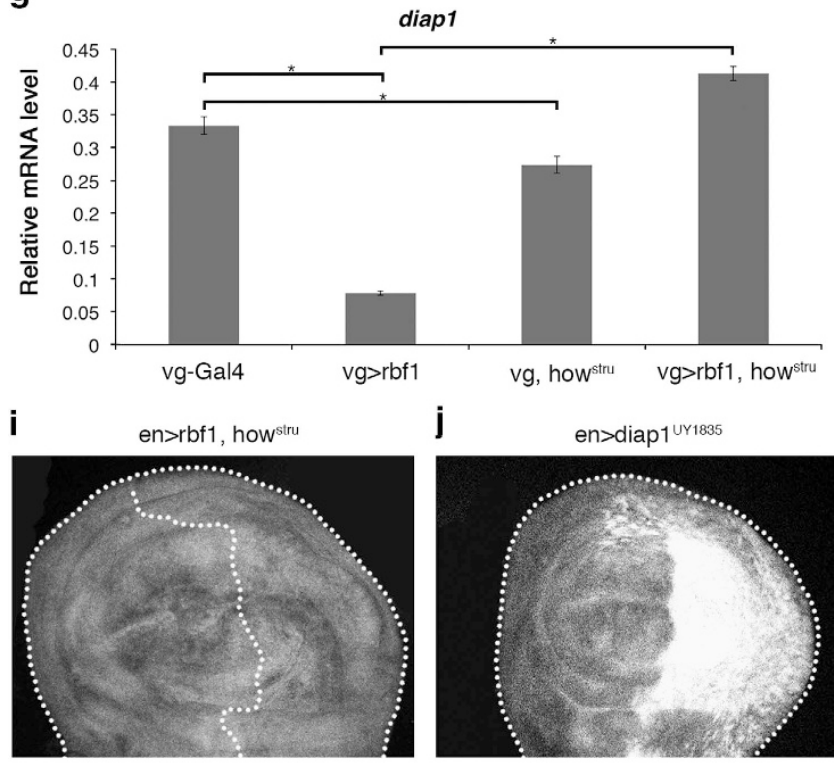

j
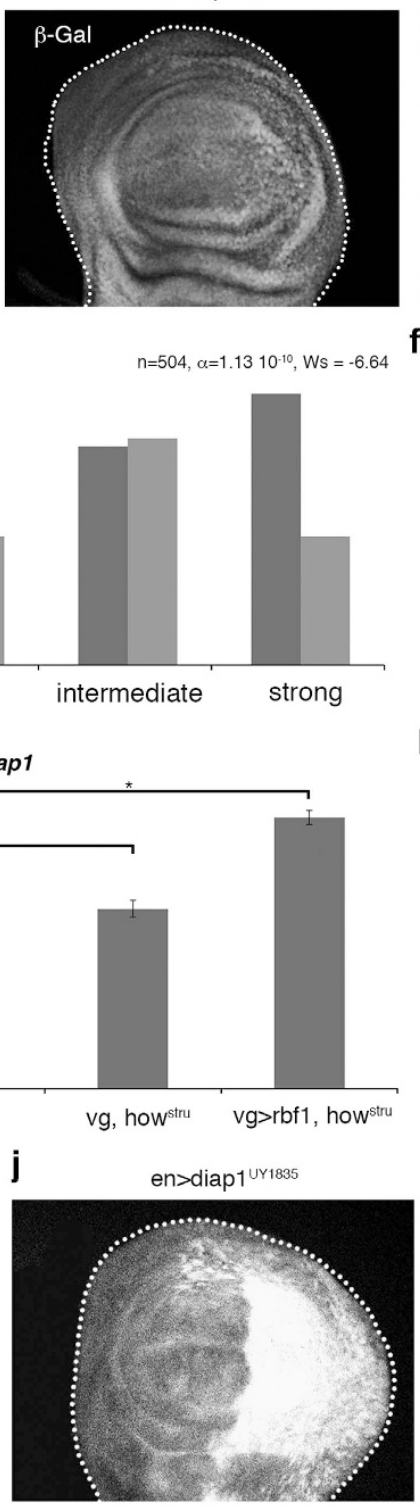

C

Diap1

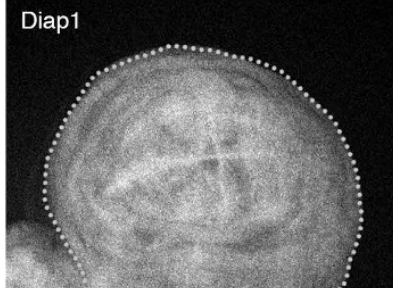

f

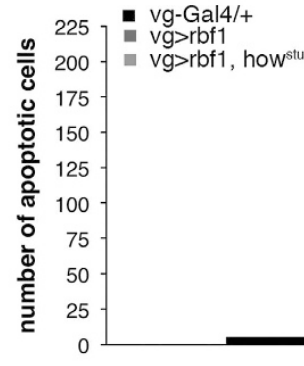

h

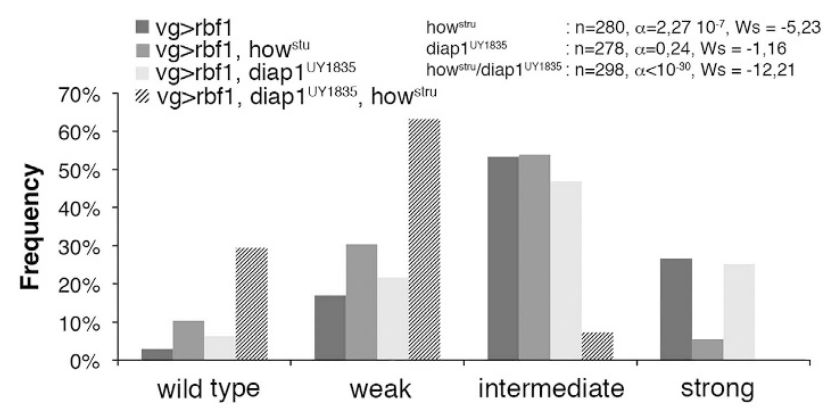

k

en>rbf1, diap $1^{\text {ur1835 }}$

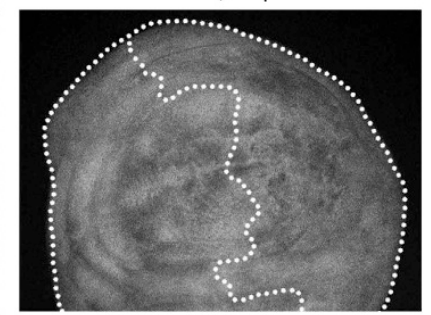

I en>rbf1, diap1 ${ }^{\text {Uy } 1835}$, how stru m

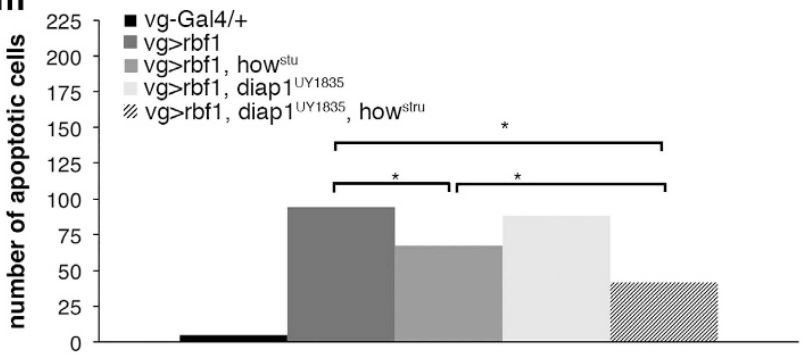

n

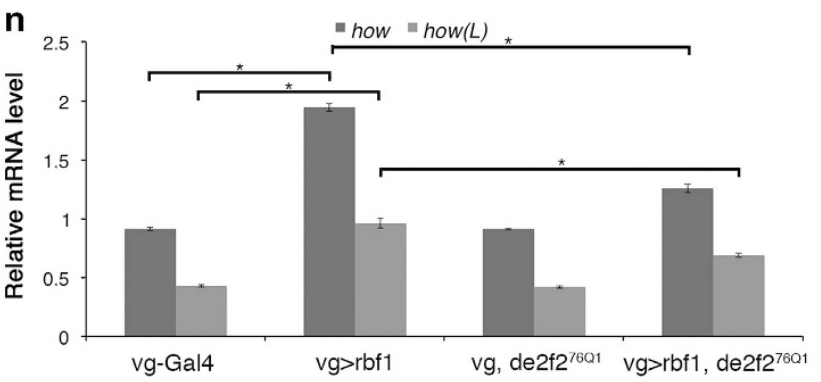

Figure 3 Rbf1 and dE2F2 increases how mRNA leading to diap1 mRNA destabilization. (a) diap1-lacZ transgene was used to report diap1 transcription in en-gal4/ + and en-gal4 > UAS-rbf1 genetic contexts. $\beta$-Gal immunostaining (white) of the discs are shown in (a, b). Diap1 immunostaining (white) are shown in (c, $\mathbf{d})$ and (i-I). The genotypes are indicated at the top of the image. Posterior compartment of the wing disc (on the right) is delimitated by dotted line when rbf1 is overexpressed. All the pictures presented in

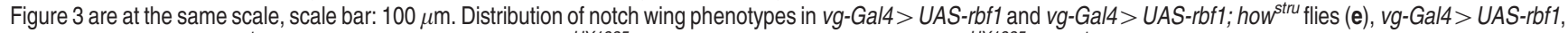
vg-Gal4 > UAS-rbf1; how ${ }^{\text {stru }}$, vg-Gal4> UAS-rbf1; UAS-diap $1^{\text {UY } 1835}$ and vg-Gal4> UAS-rbf1; UAS-diap $1^{\text {UY 1835; }}$ how ${ }^{\text {stru flies }}$ (h). Wing phenotypes were grouped into four categories according to the number of notches (wild type, weak, intermediate, strong). Statistical analysis was performed using Wilcoxon tests. Each experiment was independently performed three times; as the results were similar, only one experiment is presented here. Quantification of TUNEL-positive cells (f and $\mathbf{m}$ ) in the wing pouch of genotypes studied in (e) and (h). Asterisks indicate a statistically significant difference between two genotypes (Student's t-test, $P<0.05)$. Quantification of diap1 ( $\mathbf{g}$ ), how and how $(L)(\mathbf{n})$ mRNAs in wing imaginal discs by RT-qPCR. Data are normalized against $r p 49$ and correspond to the mean of three independent experiments. Error bars are the S.E.M. Asterisks indicate statistically significant difference between two genotypes (Student's t-test, $P<0.05$ ) 
Rbf1. These results indicate that diap1 downregulation by Rbf1 requires both the diap1 $3^{\prime} U T R$ sequence and How. We next asked whether rbf1 overexpression could affect the expression of how $(L)$. Indeed, how $(L)$ mRNA increased on rbf1 overexpression (Figure 3n). Surprisingly, this raise depended on dE2F2 as it was reduced in a de2f2 $2^{76 Q 1}$ heterozygous context. This could be explained by an indirect effect of dE2F2, or by an unusual transcriptional activity of dE2F2. Thus, these data suggest that Rbf1 and dE2F2 increase of how $(L)$ mRNA leads to destabilization of diap1 mRNA that induces apoptosis.

Rbf1 and dE2F2 binds buffy and how genomic regions near the TSSs. As the $5^{\prime}$ UTR of buffy contains dE2F2 binding sites, we performed chromatin immunoprecipitation (ChIP) experiments to determine whether Rbf1 and dE2F2 directly bound this region. We used pcna, a well-characterized target of Rbf1/dE2F complexes, as a positive control and slp1, a nontarget gene of Rbf1, ${ }^{39}$ as a negative control. No significant enrichment was observed of the negative control slp1 after Rbf1 or dE2F2 immunoprecipitation (IP). In contrast, we detected a significant enrichment of the positive control pcna promoter region on Rbf1 or dE2F2 IP as compared with the mock IP (Figures $4 \mathrm{a}$ and b). The buffy 5'UTR was significantly enriched after Rbf1 and dE2F2 IPs as compared with the negative controls both in rbf1overexpressing wing discs and control discs (Figures $4 \mathrm{a}$ and b). Therefore, Rbf1 and dE2F2 bound buffy in the wing imaginal discs. Then, Rbf1 and dE2F2 might directly repress buffy transcription.

The how promoter also contains a putative dE2F binding site suggesting that Rbf1 and dE2F2 could bind this region. Consistently, we observed that how genomic region was significantly enriched after both Rbf1 and dE2F2 IP in rbf1overexpressing wing discs (Figures $4 \mathrm{a}$ and b). Nevertheless, these enrichments were not observed with the control discs. Despite the fact that Rbf1/dE2F2 complex is almost exclusively described as a transcriptional repressor, our results suggest that Rbf1 and dE2F2 might directly activate how transcription.
Transcriptional control by different members of the dREAM complex depends on the target gene. Rbf1 and dE2F2 belong to a multi-subunit complex named dREAM. This complex positively regulates some target genes, whereas it negatively regulates some others. Certain proteins of the complex are activators, whereas others are repressors or not involved. Moreover the role of each protein can change according to the target gene. To determine the function of the dREAM subunits Mip120, Mip130 and Myb in Rbf1-induced apoptosis, we performed genetic interaction tests. When rbf1 was co-overexpressed with either RNAimip 120 or RNAi-mip130, the distribution of wing phenotypes shifted toward weaker phenotypes as compared with the expression of rbf1 alone (Figures $5 a$ and b). Consistently, the number of apoptotic cells decreased in the wing discs of the same genotypes (Figures $5 d$ and e), indicating that Mip120 and Mip130 were necessary for Rbf1-induced apoptosis.

rbf1 and myb co-expression induces a shift in the phenotype distribution toward weaker phenotypes (Figure $5 c$ ) and a decrease of apoptotic cells (Figure 5f). Therefore, Myb is sufficient to inhibit Rbf1-induced apoptosis. This result is in an agreement with the antagonistic role of Myb as compared with the role of other members of the complex, previously described. ${ }^{30}$

We next assessed the contribution of Mip120, Mip130 and Myb to buffy and how transcriptional regulation by RT-qPCR. Depletion of Mip120 or Mip130 by RNAiinduced an increase of buffy mRNA as compared with vg-Gal4/+ control (Figure $5 \mathrm{~g}$ ), indicating that Mip120 and Mip130 are required to repress buffy expression in the wing imaginal discs. Moreover, when rbf1 was co-overexpressed with RNAi-mip120, buffy mRNA increased as compared with the rbf1 expression alone. Similar results were observed with $R N A i-m i p 130$, indicating that these two genes are required for Rbf1 to repress buffy expression. Depletion of Myb by RNAi did not affect buffy expression, whereas myb overexpression increased it (Figure 5h). Furthermore, when rbf1 and myb were co-overexpressed, the amount of buffy mRNA was similar to the one of $v g$-Gal4/+ control. Thus, Myb and Mip proteins had opposite roles on buffy a
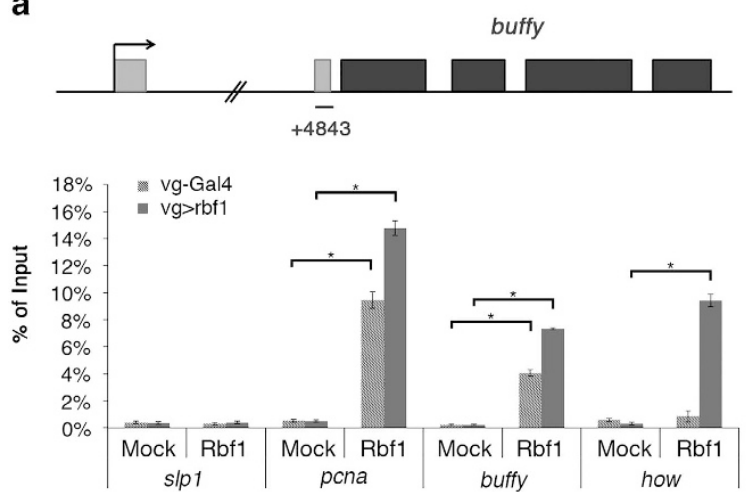

b
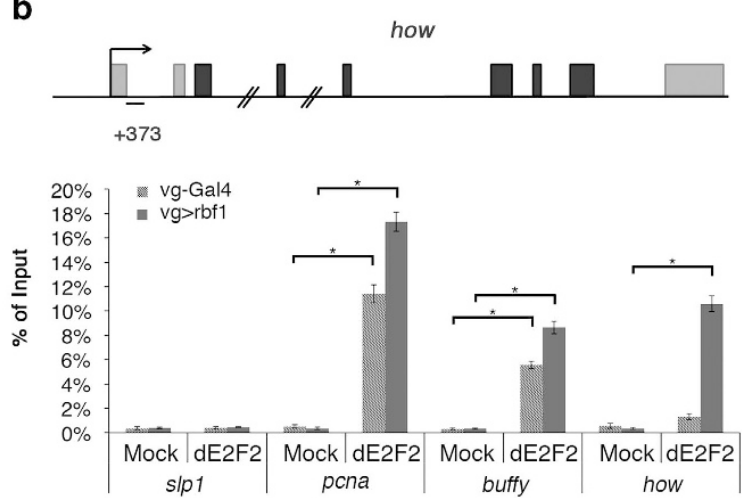

Figure 4 Binding of Rbf1 and dE2F2 on buffy and how genes. Structure of buffy (a) and how (b) genes. qPCR amplicons are indicated. Coordinated of qPCR amplicons relative to TSS are indicated. ChIP-qPCR analysis of buffy (a) and how (b) from vg-gal4 > UAS-Rbf1 or control (vg-Gal4/+) wing imaginal discs using mock, anti-Rbf1 or antidE2F2 antibodies. The results were expressed as percentages of input. slp1, a nontarget gene of Rbf1, was used as a negative control whereas pcna, a known dE2Fs target, was used as a positive control. Error bars represent S.E.M obtained from three independent experiments. Asterisks indicate statistically significant difference between two genotypes (Student's $t$-test, $P<0.05$ ) 
a

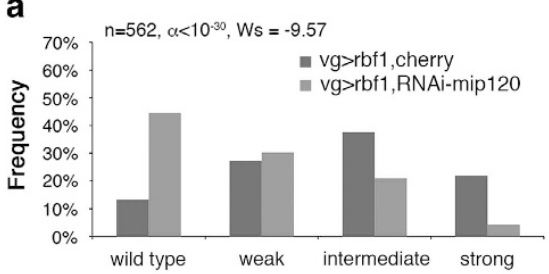

b

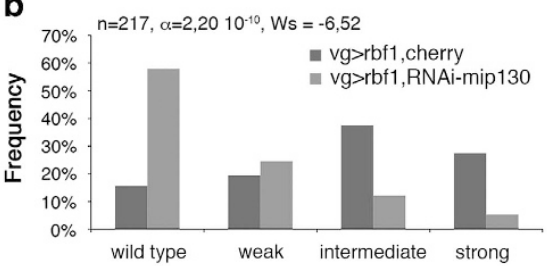

e
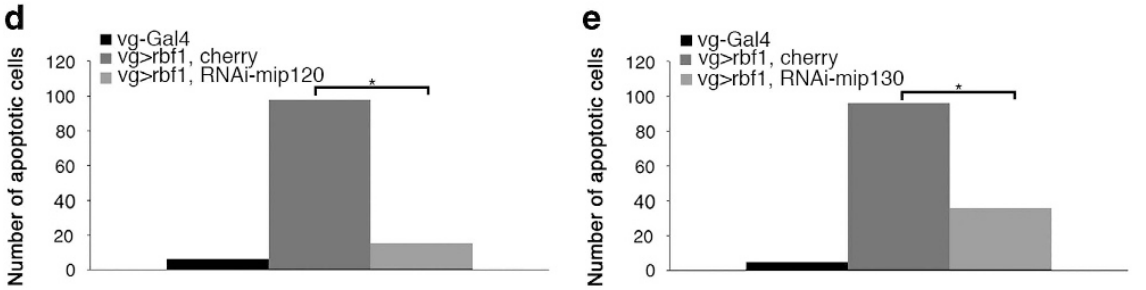

C

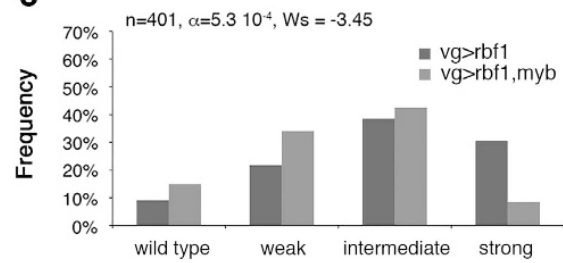

f

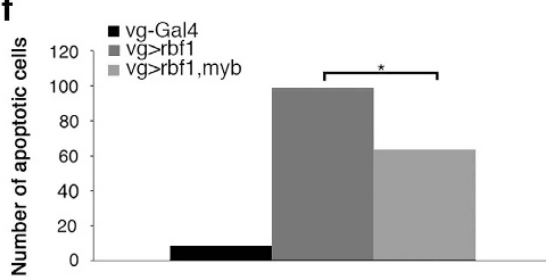

g

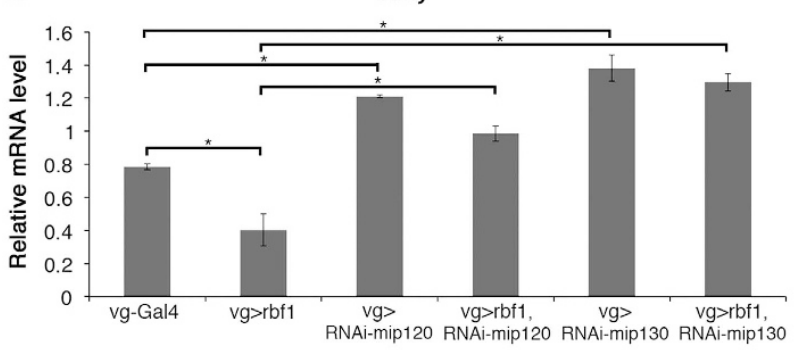

i

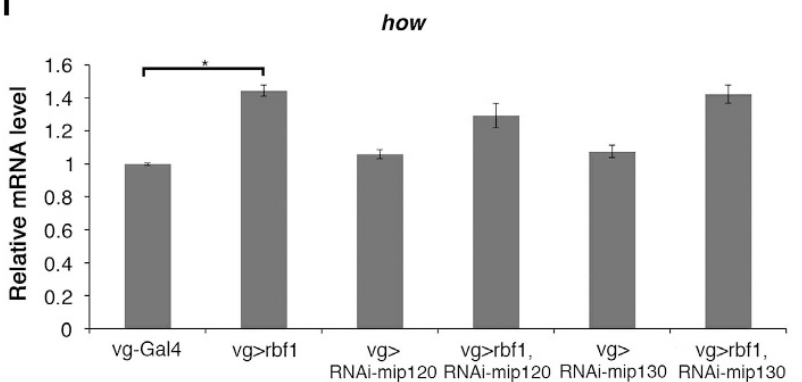

h

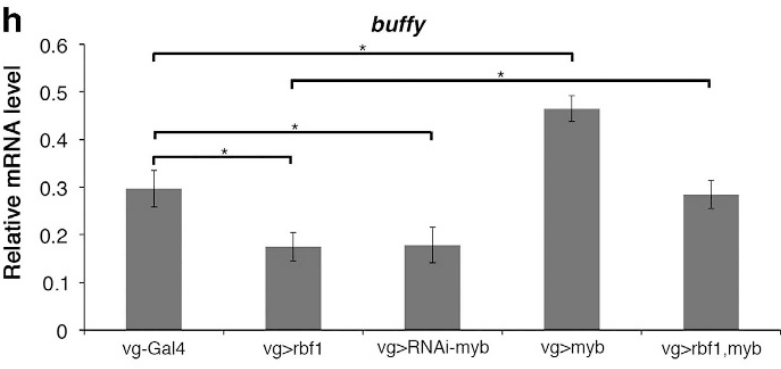

j

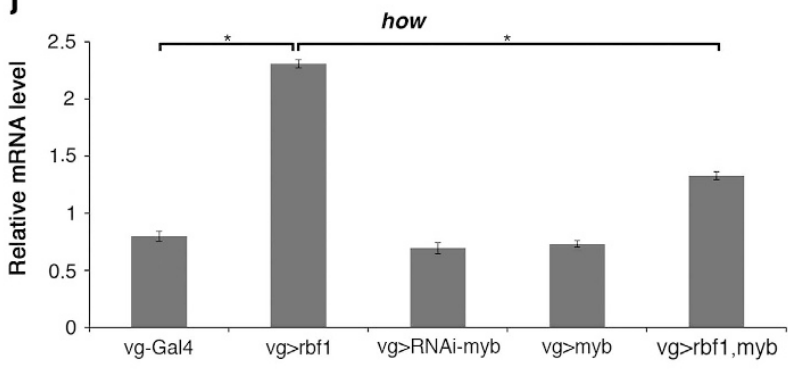

Figure 5 Mip proteins and Myb are differentially involved in Rbf1-induced apoptosis. (a-c) Distribution of notch wing phenotypes in vg-Gal4 $>$ UAS-rbf1,UAS-cherry and vg-Gal4 > UAS-rbf1,UAS-RNAi-mip120 (a), in vg-Gal4> UAS-rbf1,UAS-cherry and vg-Gal4> UAS-rbf1; UAS-RNAi-mip130 (b) and in vg-Gal4> UAS-rbf1 and $v g$-Gal4 > UAS-rbf1; UAS-myb (c). Wing phenotypes were grouped in four categories according to the number of notches (wild type, weak, intermediate, strong). Statistical analysis was performed using Wilcoxon tests. Each experiment was independently performed three times; as the results were similar, only one experiment is presented here. (d-f) Quantification of TUNEL-positive cells in the wing pouch of genotypes studied in (a-c). Asterisks indicate a statistically significant difference between two genotypes (Student's t-test, $P<0.05)$. (g-j) Quantification of buffy $(\mathbf{g}, \mathbf{h})$ and how $(\mathbf{i}, \mathbf{j})$ mRNA in wing imaginal discs by RT-qPCR. Data are normalized against $r p 49$ and correspond to the mean of three independent experiments. Error bars are the S.E.M. Asterisks indicate statistically significant difference between two genotypes (Student's $t$-test, $P<0.05)$

transcriptional regulation: whereas Myb activated buffy transcription, Mip factors repressed it.

Inactivation of Mip120 or Mip130 by RNAi did not affect the basal level of how mRNA (Figure 5i). Moreover, when rbf1 was co-overexpressed with either RNAi-mip120 or RNAi-mip130, the amount of how mRNA was similar to the one observed for rbf1 overexpression alone, suggesting that these Mip factors are not involved in the transcriptional regulation of how by Rbf1.

Inactivation or overexpression of myb did not alter the basal amount of how mRNA (Figure 5j). However, rbf1 and myb cooverexpression decreased the level of how mRNA as compared with the rbf1 overexpression alone. These data suggest that, contrary to dE2F2, Myb antagonizes Rbf1induced how transcriptional activation.

\section{Discussion}

E2F transcription factors are the main partners of Rbf1. dE2F1 is widely described for its important role in the control of cell proliferation by Rbf1, whereas the best-described role of dE2F2 is the repression of replication during oogenesis. ${ }^{35,40}$ Here, we show that dE2F2 and dDP are required for Rbf1-induced apoptosis suggesting that they are pro-apoptotic factors, contrary to dE2F1, which inhibits this apoptosis. Until now, dE2F1 was mainly described as a pro-apoptotic factor, whereas dE2F2 was most of the time described as an antiapoptotic factor. Indeed, de2f1 and $d D p$ co-expression induces apoptosis in the eye imaginal discs ${ }^{23}$ and $\mathrm{dE} 2 \mathrm{~F} 1$ promotes irradiation-induced Dp53-independent apoptosis in wing 
imaginal discs, whereas dE2F2 inhibits apoptosis in the same model. ${ }^{41}$ Recently, Rovani et al. ${ }^{42}$ showed that the dREAM complex, which includes dE2F2, cooperates with the proapoptotic factor Grim to induce cell death in the peripheral nervous system. This result suggests a pro-apoptotic role for dE2F2, which is consistent with our results. Furthermore, the role of dE2F1 in apoptosis might depend on the cellular context. Indeed, dE2F1 is important for DNA damage-induced apoptosis in the wing imaginal discs, but its role varies depending on the position of the cell within the disc. ${ }^{43}$ Together, this result and ours indicate that the role of both dE2F1 and dE2F2 in apoptosis control depends on the cellular context. Similarly, depending on the cells or tissues, Rbf1 has a pro- or antiapoptotic effect. Indeed, RBF expression induces apoptosis in different proliferative tissues, whereas this effect was not observed in differentiated post-mitotic cells. ${ }^{26}$

RNAi-based studies suggested a requirement of buffy for cell survival during embryonic development. ${ }^{44}$ Another study suggests that Buffy is not involved in developmental cell death but modulates the response to irradiation-induced cell death. ${ }^{45}$ Here, we show that buffy is involved in apoptosis induced by overexpression of the tumor suppressor gene rbf1. Furthermore, our data reveal for the first time that Rbf1 regulates buffy transcription. It would thus be interesting to determine whether Rbf1 also regulates buffy in response to irradiation.

At least in some cancers, pRb oncosuppressor activity relies on its apoptosis-inducing activity. It has been suggested that RB mutations can affect the sensitivity to mitomycin/ anthracycline treatment in breast cancer. ${ }^{46}$ Several reports underline the importance of $\mathrm{pRb}$ in the apoptotic response of prostate cancer cells to radiotherapy or chemotherapeutic drugs. ${ }^{11,12,47}$ How pRb mediates apoptosis in these cases remains unclear. Other data show that DNA damage promotes the formation of a pRB/E2F1 complex involved in the activation of pro-apoptotic genes such as Caspase 7 and $p 73,{ }^{13}$ and that RB/E2F-1 is a major contributor of Noxa induction in response to $\mathrm{ABT}-737$ treatment, a Bcl-2 inhibitor. ${ }^{48}$ Thus, pRb/E2F proapoptotic signaling(s) can be activated in response to oncogenic stress, DNA damage or Bcl-2 inhibition. Similarly, our data show that in Drosophila, Rbf1/dE2F2 can regulate apoptosis by upregulating how (Figure 6). Although this regulation seems non-essential to maintain the how mRNA basal level, we cannot exclude that it occurs in response to some stresses and/or therapeutic treatments. Indeed, Quaking, the homolog of how, has been shown to be a tumor suppressor. ${ }^{49-51}$ It would be interesting to study whether a pRb/E2F complex can regulate a mammalian homolog of the dIAP1 gene via Quaking.

Rbf1 and dE2F2 belong to a large complex called dREAM. Inactivation of members of dREAM by RNAi in Kc cells leads to variations of how and buffy expression, ${ }^{32}$ which suggests that these two genes could be direct transcriptional targets of dREAM. Such as described by Georlette et al., ${ }^{32}$ we found that the involvement of dREAM complex members are not equivalent: some members can be activators for a specific target, whereas others are repressors or are not involved in transcriptional regulation of this target. Indeed dE2F2, Mip120 and Mip130 are required for Rbf1-induced transcriptional repression of buffy, whereas Myb has an opposite effect. Moreover, contrary to dE2F2 and Myb, Mip120 and Mip130 are not involved in how regulation.

dE2F2 and Myb have opposite effects on Rbf1-induced buffy and how transcription. Our results are in agreement with transcriptomic data indicating that there are no genes negatively co-regulated by Myb and dE2F2 but many genes are regulated both positively by Myb and negatively by dE2F2. ${ }^{32}$

Our results confirm a previous report ${ }^{32}$ indicating that $\mathrm{Rbf} 1$ and the dREAM complex can act both as a transcriptional activator and as a transcriptional repressor (Figure 6). The molecular mechanisms for transcriptional repression have

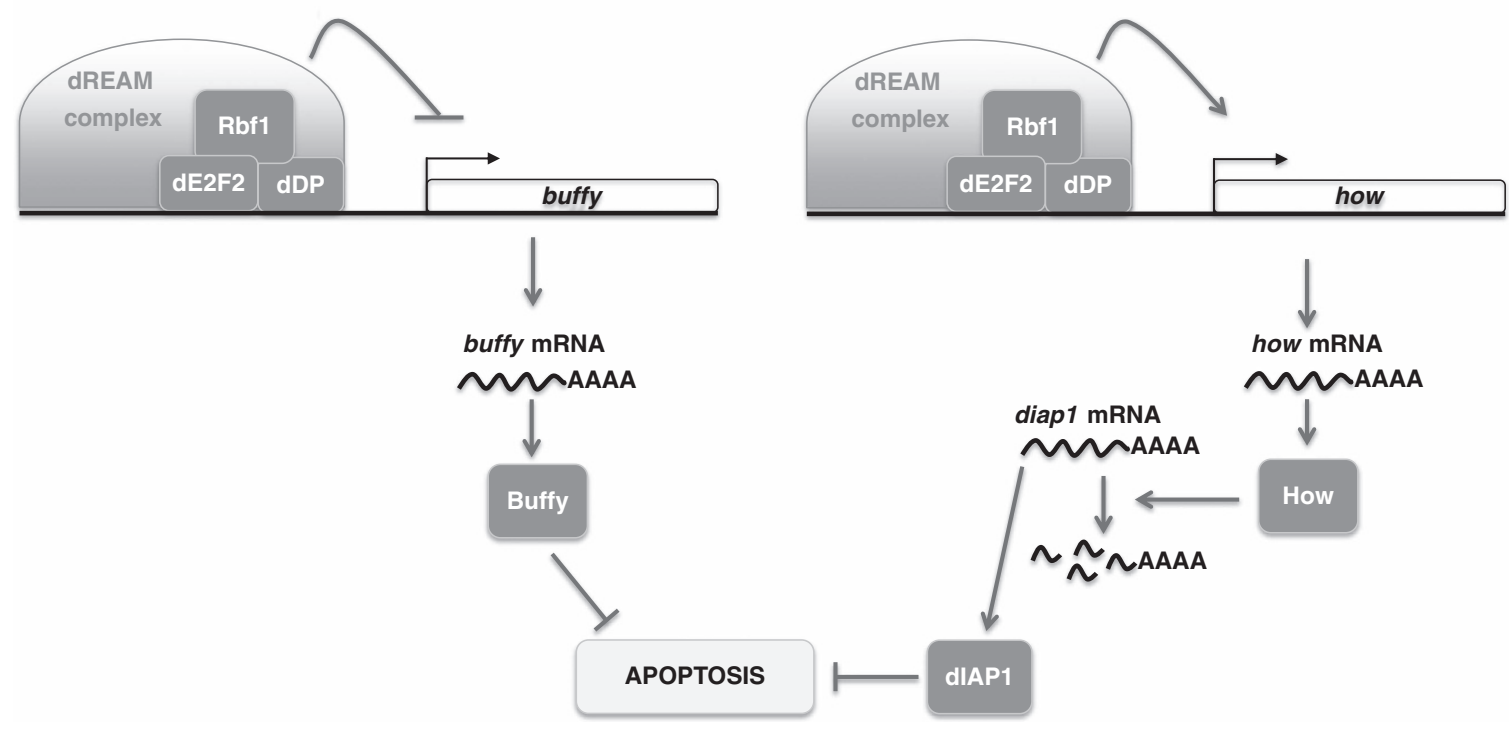

Figure 6 Rbf1-induced apoptosis involved transcriptional regulation of buffy and how genes. Rbf1 with dE2F2 binds buffy genomic site near to TSS and represses its transcription. The decrease of buffy mRNA contributes to Rbf1-induced apoptosis. Rbf1 can also bind how promoter with dE2F2 but this time it induces a transcriptional upregulation. As a consequence, How level increases allowing diap1 mRNA degradation which promotes Rbf1-induced cell death. Some members of the dREAM complex are involved in these transcriptional regulations 
been deciphered. ${ }^{31}$ It would be interesting to determine which epigenetic mechanisms are involved in the transcriptional activation of dREAM complex target genes.

Using an overexpression system, we have identified the bcl-2 family gene buffy, as a transcriptional target of Rbf1. In an rbf1 loss-of-function mutant, buffy transcription increases show that in wild-type cells not committed to apoptosis, Rbf1 is necessary to limit buffy expression. Thus, rbf1 loss of function could render cells more resistant to apoptosis. Identification of buffy as an Rbf1/dE2F pathway target gene is consistent with the role of tumor suppressor described for the human counterpart $\mathrm{Rb}$. Transcriptional regulation of $b c /-2$ family genes by $\mathrm{Rb} / \mathrm{E} 2 \mathrm{~F}$ complexes has also been characterized in mammals. Indeed, pRb/E2F1 directly regulates noxa, ${ }^{48}$ bim $^{52}$ and puma. ${ }^{53}$ Regulation by $\mathrm{Rb}$ of $\mathrm{bcl}-2$ family gene expression may have a major impact on cell death and can thus contribute to its tumor suppressor action.

\section{Materials and Methods}

Fly stocks. Flies were raised at $25^{\circ} \mathrm{C}$ on a standard medium. The UAS-Rbf1 and $v g$-Gal4 strains were generous gifts from $\mathrm{J}$ Silber. The en-Gal4 strain was kindly provided by $L$ Théodore. The UAS-diap1 strain was a generous gift from A-M Pret. In this strain, a transgene containing diap1 CDNA under the control of a UAS sequence is inserted on the second chromosome and allows the expression of diap1 without its $3^{\prime}$ UTR sequence. The UAS-diap $1^{U Y 1835}$ was a kind gift from S Netter. ${ }^{38}$ In this transgenic line, a $P$ element is inserted in the $5^{\prime}$ UTR sequence of diap1 gene in the correct orientation to allow the expression of diap1 with its $3^{\prime}$ UTR sequence. The following strains were obtained from the Bloomington Stock Center (Bloomington, IN, USA): dE2F2 ${ }^{76 Q 1}$ (7436), UAS-dE2F2 (17314), dDp $p^{a 1}$ (7277), dE2F1' ${ }^{12}$ (7274), buffy ${ }^{\mathrm{H} 37}$ (27340), UAS-buffy (32059), diap1-lacZ (12093), how $^{\text {stru }}$ (2301), UAS-RNAi-mip120 (32461), UAS-RNAi-mip130 (32462), UAS-RNAimyb (35053), UAS-myb (32044). The UAS-RNAi-diap1 strain was from NIG collection (12284R-2). The UAS-RNAi-rbf1 strain was from VDRC collection (10696).

Test of phenotype suppression in the wing. To test the implication of several genes (dE2F1, dE2F2, dDP, Buffy, Diap1, How, Mip120, Mip130 and Myb) in $r b f 1$-induced apoptosis, the severity of the notched wing phenotype induced by UAS-Rbf1 overexpression led by $\mathrm{vg}$-Gal4 driver was assayed in different genetic contexts. For each gene, we verified that the alteration of this gene expression level (overexpression, RNAi or mutant) did not induce any wing phenotype. $v g$ Gal4 > UAS-Rbf1 Drosophila females were crossed with males bearing a loss-offunction mutation for the different genes or allowing their overexpression. The progenies of all crosses were classified according to the number of notches on the wing margin. Wilcoxon tests were performed as described previously. ${ }^{54}$

TUNEL staining of imaginal discs. Third instar larvae were dissected in PBS pH 7.6, fixed in PBS/formaldehyde 3.7\%, washed three times for 10 min in PBT $(1 \times$ PBS, $0.5 \%$ Triton). Discs were then dissected and TUNEL staining was performed according to manufacturer's instructions (ApopTag Red in situ apoptosis detection kit, Millipore, Temecula, CA, USA). Discs were mounted in CitifluorTM (Biovalley, Marne-La-Vallée, France) and observed with a Leica SPE upright confocal microscope (Leica, Wetzlar, Germany). White patches in the wing pouch were counted for at least 30 wing imaginal discs per genotype. Student's $t$-tests were performed and results were considered to be significant when $\alpha<5 \%$.

Immunochemistry. The following antibodies were used: anti- $\beta$-Gal (mouse monoclonal antibody, 1/200, 40-1a, DSHB) and anti-Diap1 (mouse monoclonal antibody, $1 / 200$, generous gift from B Hay). Third instar larvae were dissected in PBS pH 7.6, fixed in PBS-3.7\% formaldehyde, washed three times for 10 min each in PBT (PBS, $0,3 \%$ Triton) and incubated with primary antibody overnight at $4{ }^{\circ} \mathrm{C}$ in PBT-FCS (PBS, 0,3\% Triton, 10\% FCS). Incubation with anti-mouse secondary antibody (Alexa Fluor 488 Goat Anti-Mouse IgG $(\mathrm{H}+\mathrm{L})$ Antibody, Molecular Probes, Thermo Fisher Scientific, Waltham, MA, USA) was carried in PBT-FCS for $2 \mathrm{~h}$ at room temperature. Larvae were then washed thrice in PBT. Finally, wing discs were mounted in CitifluorTM (Biovalley) and observed with a Leica SPE upright confocal microscope.
Chromatin immunoprecipitation. ChIPs were performed as previously described, ${ }^{55}$ with minor modification. Briefly, $50 \mathrm{vg}$-Gal4> UAS-rbf1 wing imaginal discs of third instar larvae were dissected on ice in serum-free Schneider medium. They were fixed in $500 \mu \mathrm{l}$ of formaldehyde (1.8\% in PBS) for $10 \mathrm{~min}$ at room temperature under gentle agitation. Cross-linking was stopped by adding $50 \mu \mathrm{l}$ of glycine 1.25 M. Fixed wing discs were washed 3 times with PBS, dried, flash-freezed in liquid nitrogen and stored at $-80^{\circ} \mathrm{C}$. Cell lysis was performed by adding $100 \mu \mathrm{l}$ of lysis buffer $(140 \mathrm{mM} \mathrm{NaCl}, 10 \mathrm{mM}$ Tris-HCl pH 8.0, $1 \mathrm{mM}$ EDTA, $1 \%$ Triton X-100, $0.1 \%$ sodium deoxycholate, Roche complete EDTA-free protease inhibitor cocktail) complemented with $1 \%$ SDS and sonicated in a Bioruptor sonifier (Diagenode, Seraing, Belgium). Conditions were established to obtain chromatin fragments from 200 to $1000 \mathrm{bp}$ in length (30 s ON 30 s OFF, high power, 10 cycles). Pooled chromatin was centrifuged for $20 \mathrm{~min}$ at $14000 \mathrm{~g}$ at $4^{\circ} \mathrm{C}$. The supernatant (soluble chromatin) was recovered and $10 \mu \mathrm{l}$ were kept as input sample. For each IP, $10 \mu \mathrm{l}$ of protein A-coated paramagnetic beads (Diagenode) were washed once in lysis buffer, $1 \mu \mathrm{g}$ of antibody was added and beads were incubated for $2 \mathrm{~h}$ at $4{ }^{\circ} \mathrm{C}$ on a rotating wheel. After washing in lysis buffer, antibody coated beads were resuspended in $300 \mu \mathrm{l}$ of lysis buffer and $100 \mu \mathrm{l}$ of chromatin were added. After incubation on a rotating wheel overnight at $4^{\circ} \mathrm{C}$, beads were washed at $4{ }^{\circ} \mathrm{C}$ five times for 10 min each in lysis buffer, once in $\mathrm{LiCl}$ buffer (Tris- $\mathrm{HCl} 10 \mathrm{mM} \mathrm{pH8.0,} \mathrm{LiCl}$ $0.25 \mathrm{M}, 0.5 \% \mathrm{NP}-40,0.5 \%$ sodium deoxycholate, $1 \mathrm{mM}$ EDTA) and twice in TE (10 mM Tris-HCl, pH 8.0, $1 \mathrm{mM}$ EDTA). Immunoprecipitated as well as input DNAs were purified with the IPure kit following the manufacturer's instructions (Diagenode). Elution was performed twice with $35 \mu \mathrm{l}$ of water. $5 \mu \mathrm{l}$ of DNA were used per PCR. Real-time PCR data were normalized against the input sample and depicted as percentage of input (see Supplementary Table S1 for primers). pcna, a well characterized target of Rbf1/dE2F complexes, was used as a positive control and $s / p 1$, a nontarget gene of Rbf1, ${ }^{39}$ as a negative control.

Antibodies used for chromatin immunoprecipitation were anti-Rbf1 (rabbit polyclonal, Custom antibody against amino acids 250-845 of Rbf1 protein, Proteogenix, Schiltigheim, France), anti-dE2F2 (rabbit polyclonal, Custom antibody against the whole protein, Proteogenix). Rabbit pre-immune sera were used as negative controls (mocks).

RNAs extraction and RT-qPCR. Fifty wing imaginal discs per genotype were dissected on ice in serum-free Schneider medium. Total RNAs were extracted from each sample using the RNeasy Mini kit (Qiagen, Venlo, Netherlands), by following the manufacturer's instructions. RT was performed on each sample using $4.8 \mu \mathrm{g}$ of RNA incubated with random primer oligonucleotides (Invitrogen, Life Technologies, Carlsbad, CA, USA) with Recombinant Taq DNA Polymerase (Invitrogen), according to the manufacturer's instructions.

Real-time PCR analysis was performed using the ABI Prism $7700 \mathrm{HT}$ apparatus (Applied Biosystems, Life Technologies). Briefly, PCR was performed with the ABsolute blue QPCR SYBR Green ROX mix (Abgene, Thermo Fisher Scientific), using $11 \mathrm{ng}$ of cDNA per RT. The primers used for real-time PCR are presented in Supplementary Table S2. Data were normalized against $r p 49$. Three independent RT experiments were performed and the S.E.M was calculated from these three independent samples.

\section{Conflict of Interest}

The authors declare no conflict of interest.

Acknowledgements. We are grateful to S Szuplewski, S Gaumer and F Peronnet for their critical reading of the manuscript. We thank B Hay for a generous gift of dIAP1 antibody. Confocal microscopy was performed on CYMAGES imaging facility. qPCR experiments were done in the UMR 1198 'Biologie du Développement et Reproduction' (INRA, Jouy-en-Josas). This work was supported by the 'Université de Versailles Saint-Quentin-en-Yvelines' (UVSQ) and by grants from the 'Ligue Nationale Contre le Cancer'. Amandine Clavier was the recipient of a doctoral contract from UVSQ. Adrienne Baillet was supported by the UVSQ and the Ecole Pratique des Hautes Etudes.

1. van den Heuvel S, Dyson NJ. Conserved functions of the pRB and E2F families. Nat Rev $\mathrm{Mol}$ Cell Biol 2008; 9: 713-724.

2. Frolov MV, Dyson NJ. Molecular mechanisms of E2F-dependent activation and pRB-mediated repression. J Cell Sci 2004; 117: 2173-2181. 
3. Almasan A, Yin Y, Kelly RE, Lee EY, Bradley A, Li W et al. Deficiency of retinoblastoma protein leads to inappropriate S-phase entry, activation of E2F-responsive genes, and apoptosis. Proc Natl Acad Sci USA 1995; 92: 5436-5440.

4. Knudsen KE, Booth D, Naderi S, Sever-Chroneos Z, Fribourg AF, Hunton IC et al. RB-dependent S-phase response to DNA damage. Mol Cell Biol 2000; 20: 7751-7763.

5. Clarke AR, Maandag ER, van Roon M, van der Lugt NM, van der Valk M, Hooper ML et al. Requirement for a functional Rb-1 gene in murine development. Nature 1992; 359: 328-330.

6. Jacks T, Fazeli A, Schmitt EM, Bronson RT, Goodell MA, Weinberg RA. Effects of an Rb mutation in the mouse. Nature 1992; 359: 295-300.

7. Lee EY, Chang CY, Hu N, Wang YC, Lai CC, Herrup K et al. Mice deficient for Rb are nonviable and show defects in neurogenesis and haematopoiesis. Nature 1992; 359: 288-294.

8. Tsai KY, Hu Y, Macleod KF, Crowley D, Yamasaki L, Jacks T. Mutation of E2f-1 suppresses apoptosis and inappropriate $\mathrm{S}$ phase entry and extends survival of $\mathrm{Rb}$-deficient mouse embryos. Mol Cell 1998; 2: 293-304.

9. Huh MS, Parker MH, Scime A, Parks R, Rudnicki MA. Rb is required for progression through myogenic differentiation but not maintenance of terminal differentiation. J Cell Biol 2004; 166: 865-876.

10. Biasoli D, Kahn SA, Cornelio TA, Furtado M, Campanati L, Chneiweiss $\mathrm{H}$ et al. Retinoblastoma protein regulates the crosstalk between autophagy and apoptosis, and favors glioblastoma resistance to etoposide. Cell Death Dis 2013; 4: e767.

11. Zhao X, Day ML. RB activation and repression of C-MYC transcription precede apoptosis of human prostate epithelial cells. Urology 2001; 57: 860-865.

12. Bowen C, Spiegel S, Gelmann EP. Radiation-induced apoptosis mediated by retinoblastoma protein. Cancer Res 1998; 58: 3275-3281.

13. lanari $A$, Natale $T$, Calo E, Ferretti E, Alesse E, Screpanti I et al. Proapoptotic function of the retinoblastoma tumor suppressor protein. Cancer Cell 2009; 15: 184-194.

14. Hilgendorf KI, Leshchiner ES, Nedelcu S, Maynard MA, Calo E, lanari A et al. The retinoblastoma protein induces apoptosis directly at the mitochondria. Genes Dev 2013; 27: 1003-1015.

15. Dynlacht BD, Brook A, Dembski M, Yenush L, Dyson N. DNA-binding and trans-activation properties of Drosophila E2F and DP proteins. Proc Natl Acad Sci USA 1994; 91: 6359-6363.

16. Ohtani K, Nevins JR. Functional properties of a Drosophila homolog of the E2F1 gene. Mol Cell Biol 1994; 14: 1603-1612.

17. Sawado T, Yamaguchi M, Nishimoto Y, Ohno K, Sakaguchi K, Matsukage A. dE2F2, a novel E2F-family transcription factor in Drosophila melanogaster. Biochem Biophys Res Commun 1998; 251: 409-415.

18. Du W, Vidal M, Xie JE, Dyson N. RBF, a novel RB-related gene that regulates E2F activity and interacts with cyclin E in Drosophila. Genes Dev 1996; 10: 1206-1218.

19. Stevaux O, Dimova D, Frolov MV, Taylor-Harding B, Morris E, Dyson N. Distinct mechanisms of E2F regulation by Drosophila RBF1 and RBF2. Embo J 2002; 21: 4927-4937.

20. Du W. Suppression of the rbf null mutants by a de2f1 allele that lacks transactivation domain. Development 2000; 127: 367-379.

21. Frolov MV, Huen DS, Stevaux O, Dimova D, Balczarek-Strang K, Elsdon M et al. Functional antagonism between E2F family members. Genes Dev 2001; 15: 2146-2160.

22. Du W, Dyson N. The role of RBF in the introduction of $\mathrm{G} 1$ regulation during Drosophila embryogenesis. Embo J 1999; 18: 916-925.

23. Du W, Xie JE, Dyson N. Ectopic expression of dE2F and dDP induces cell proliferation and death in the Drosophila eye. Embo J 1996; 15: 3684-3692.

24. Asano M, Nevins JR, Wharton RP. Ectopic E2F expression induces $S$ phase and apoptosis in Drosophila imaginal discs. Genes Dev 1996; 10: 1422-1432.

25. Zhou L, Steller H. Distinct pathways mediate UV-induced apoptosis in Drosophila embryos. Dev Cell 2003; 4: 599-605.

26. Milet C, Rincheval-Arnold A, Mignotte B, Guenal I. The Drosophila retinoblastoma protein induces apoptosis in proliferating but not in post-mitotic cells. Cell Cycle 2010; 9: 97-103.

27. Korenjak M, Anderssen E, Ramaswamy S, Whetstine JR, Dyson NJ. RBF binding to both canonical E2F targets and noncanonical targets depends on functional dE2F/dDP complexes. Mol Cell Biol 2012; 32: 4375-4387.

28. Korenjak M, Taylor-Harding B, Binne UK, Satterlee JS, Stevaux O, Aasland R et al. Native E2F/RBF complexes contain Myb-interacting proteins and repress transcription of developmentally controlled E2F target genes. Cell 2004; 119: 181-193.

29. Taylor-Harding B, Binne UK, Korenjak M, Brehm A, Dyson NJ. p55, the Drosophila ortholog of $\mathrm{RbAp46/RbAp48,} \mathrm{is} \mathrm{required} \mathrm{for} \mathrm{the} \mathrm{repression} \mathrm{of} \mathrm{dE2F2/RBF-regulated} \mathrm{genes.} \mathrm{Mol} \mathrm{Cell}$ Biol 2004; 24: 9124-9136.

30. Lewis PW, Beall EL, Fleischer TC, Georlette D, Link AJ, Botchan MR. Identification of a Drosophila Myb-E2F2/RBF transcriptional repressor complex. Genes Dev 2004; 18: 2929-2940.

31. Lee H, Ohno K, Voskoboynik Y, Ragusano L, Martinez A, Dimova DK. Drosophila RB proteins repress differentiation-specific genes via two different mechanisms. Mol Cell Biol 2010; 30: 2563-2577.

32. Georlette D, Ahn S, MacAlpine DM, Cheung E, Lewis PW, Beall EL et al. Genomic profiling and expression studies reveal both positive and negative activities for the Drosophila Myb MuvB/dREAM complex in proliferating cells. Genes Dev 2007; 21: 2880-2896.

33. Acharya P, Negre N, Johnston J, Wei $Y$, White KP, Henry RW et al. Evidence for autoregulation and cell signaling pathway regulation from genome-wide binding of the Drosophila retinoblastoma protein. G3 (Bethesda) 2012; 2: 1459-1472.
34. Frolov MV, Moon NS, Dyson NJ. dDP is needed for normal cell proliferation. Mol Cell Biol 2005; 25: 3027-3039.

35. Bosco G, Du W, Orr-Weaver TL. DNA replication control through interaction of E2F-RB and the origin recognition complex. Nat Cell Biol 2001; 3: 289-295.

36. Reuveny A, Elhanany $H$, Volk T. Enhanced sensitivity of midline glial cells to apoptosis is achieved by HOW(L)-dependent repression of Diap1. Mech Dev 2009; 126: 30-41.

37. Volk T, Israeli D, Nir R, Toledano-Katchalski H. Tissue development and RNA control: 'HOW' is it coordinated? Trends Genet 2008; 24: 94-101.

38. Marchal C, Vinatier G, Sanial M, Plessis A, Pret AM, Limbourg-Bouchon B et al. The HIV-1 Vpu protein induces apoptosis in Drosophila via activation of JNK signaling. PLOS One 2012; 7: e34310.

39. Acharya P, Raj N, Buckley MS, Zhang L, Duperon S, Williams G et al. Paradoxical instability-activity relationship defines a novel regulatory pathway for retinoblastoma proteins. Mol Biol Cell 2010; 21: 3890-3901.

40. Tower J. Developmental gene amplification and origin regulation. Annu Rev Genet 2004; 38: 273-304.

41. Wichmann A, Uyetake L, Su TT. E2F1 and E2F2 have opposite effects on radiationinduced p53-independent apoptosis in Drosophila. Dev Biol 2010; 346: 80-89.

42. Rovani MK, Brachmann CB, Ramsay G, Katzen AL. The dREAM/Myb-MuvB complex and Grim are key regulators of the programmed death of neural precursor cells at the Drosophila posterior wing margin. Dev Biol 2012; 372: 88-102.

43. Moon NS, Frolov MV, Kwon EJ, Di Stefano L, Dimova DK, Morris EJ et al. Drosophila E2F1 has context-specific pro- and antiapoptotic properties during development. Dev Cell 2005. 9: 463-475.

44. Quinn L, Coombe M, Mills K, Daish T, Colussi P, Kumar S et al. Buffy, a Drosophila Bcl-2 protein, has anti-apoptotic and cell cycle inhibitory functions. EMBO J 2003; 22: 3568-3579.

45. Sevrioukov EA, Burr J, Huang EW, Assi HH, Monserrate JP, Purves DC et al. Drosophila Bcl-2 proteins participate in stress-induced apoptosis, but are not required for normal development. Genesis 2007; 45: 184-193.

46. Berge EO, Knappskog S, Geisler S, Staalesen V, Pacal M, Borresen-Dale AL et al. Identification and characterization of retinoblastoma gene mutations disturbing apoptosis in human breast cancers. Mol Cancer 2010; 9: 173

47. Sharma A, Comstock CE, Knudsen ES, Cao KH, Hess-Wilson JK, Morey LM et al. Retinoblastoma tumor suppressor status is a critical determinant of therapeutic response in prostate cancer cells. Cancer Res 2007; 67: 6192-6203.

48. Bertin-Ciftci J, Barre B, Le Pen J, Maillet L, Couriaud C, Juin P et al. pRb/E2F-1-mediated caspase-dependent induction of Noxa amplifies the apoptotic effects of the Bcl-2/Bcl-xL inhibitor ABT-737. Cell Death Differ 2013; 20: 755-764.

49. Yin D, Ogawa S, Kawamata N, Tunici P, Finocchiaro G, Eoli $M$ et al. High-resolution genomic copy number profiling of glioblastoma multiforme by single nucleotide polymorphism DNA microarray. Mol Cancer Res 2009; 7: 665-677.

50. Bian Y, Wang L, Lu H, Yang G, Zhang Z, Fu H et al. Downregulation of tumor suppressor QKI in gastric cancer and its implication in cancer prognosis. Biochem Biophys Res Commun 2012; 422: 187-193

51. Zhao $Y$, Zhang G, Wei M, Lu X, Fu H, Feng F et al. The tumor suppressing effects of QKI-5 in prostate cancer: a novel diagnostic and prognostic protein. Cancer Biol Ther 2014; 15: 108-118.

52. Zhao Y, Tan J, Zhuang L, Jiang X, Liu ET, Yu Q. Inhibitors of histone deacetylases target the Rb-E2F1 pathway for apoptosis induction through activation of proapoptotic protein Bim. Proc Natl Acad Sci USA 2005; 102: 16090-16095.

53. Hao H, Dong Y, Bowling MT, Gomez-Gutierrez JG, Zhou HS, McMasters KM. E2F-1 induces melanoma cell apoptosis via PUMA up-regulation and Bax translocation. BMC Cancer 2007; 7: 24

54. Brun S, Rincheval V, Gaumer S, Mignotte B, Guenal I. reaper and bax initiate two different apoptotic pathways affecting mitochondria and antagonized by bcl-2 in Drosophila. Oncogene 2002; 21: 6458-6470.

55. Coleno-Costes A, Jang SM, de Vanssay A, Rougeot J, Bouceba T, Randsholt NB et al. New partners in regulation of gene expression: the enhancer of Trithorax and Polycomb Corto interacts with methylated ribosomal protein 112 via its chromodomain. PLoS Genet 2012; 8: e1003006.

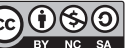

Cell Death and Disease is an open-access journal published by Nature Publishing Group. This work is licensed under a Creative Commons Attribution-NonCommercialShareAlike 3.0 Unported License. The images or other third party material in this article are included in the article's Creative Commons license, unless indicated otherwise in the credit line; if the material is not included under the Creative Commons license, users will need to obtain permission from the license holder to reproduce the material. To view a copy of this license, visit http://creativecommons.org/licenses/ by-nc-sa/3.0/ 